\title{
A note on sum-product estimates over finite valuation rings
}

\author{
Pham Duc Hiep
}

\begin{abstract}
Let $\mathcal{R}$ be a finite valuation ring of order $q^{r}$ with $q$ a power of an odd prime number, and $\mathcal{A}$ be a set in $\mathcal{R}$. In this paper, we improve a recent result due to Yazici (2018) on a sum-product
\end{abstract} type problem. More precisely, we will prove that

1. If $|\mathcal{A}| \gg q^{r-\frac{1}{3}}$, then

$$
\max \left\{|\mathcal{A}+\mathcal{A}|,\left|\mathcal{A}^{2}+\mathcal{A}^{2}\right|\right\} \gg q^{\frac{r}{2}}|\mathcal{A}|^{\frac{1}{2}}
$$

2. If $q^{r-\frac{3}{8}} \ll|\mathcal{A}| \ll q^{r-\frac{1}{3}}$, then

$$
\max \left\{|\mathcal{A}+\mathcal{A}|,\left|\mathcal{A}^{2}+\mathcal{A}^{2}\right|\right\} \gg \frac{|\mathcal{A}|^{2}}{q^{\frac{2 r-1}{2}}} .
$$

3. If $|\mathcal{A}+\mathcal{A}||\mathcal{A}|^{2} \gg q^{3 r-1}$ and $2 q^{r-1} \leq|\mathcal{A}| \ll q^{r-\frac{3}{8}}$, then

$$
\max \left\{|\mathcal{A}+\mathcal{A}|,\left|\mathcal{A}^{2}+\mathcal{A}^{2}\right|\right\} \gg q^{r / 3}|\mathcal{A}|^{2 / 3} \text {. }
$$

\section{Introduction}

Let $A$ be a subset of integers, we define the sum set and the product set, respectively, as follows

$$
\begin{gathered}
\mathcal{A}+\mathcal{A}=\{a+b: a, b \in \mathcal{A}\}, \\
\mathcal{A} \cdot \mathcal{A}=\{a \cdot b: a, b \in \mathcal{A}\} .
\end{gathered}
$$

In 1983, Erdős and Szemerédi [3] proved that there is no set $\mathcal{A}$ which is both highly additive structured and multiplicative structured at the same time. More precisely, they proved that

$$
\max \{|\mathcal{A}+\mathcal{A}|,|\mathcal{A} \cdot \mathcal{A}|\} \geq|\mathcal{A}|^{1+c}
$$

for some constant $c>0$

Over last twenty years, there was intensive progress on improving the constant $c$ and on studying variants in different settings, for example, over finite fields and the complex numbers. We refer the interested reader to [2, 7, 11, 12, 17, 18, and references therein for more details.

Let $\mathcal{R}$ be a finite valuation ring, i.e. a finite, local and principal ring. The first result on sumproduct type problems in the context of finite valuation rings was given in [6] by Ham, Pham and Vinh. In particular, they proved the following theorems.

Theorem 1.1 (Ham-Pham-Vinh, [6]). Let $\mathcal{R}$ be a finite valuation ring of order $q^{r}$, and $\mathcal{R}^{*}$ denote the set of units of $\mathcal{R}$. Let $G$ be a subgroup of $\mathcal{R}^{*}$, and $f(x, y)=g(x)(h(x)+y)$ be defined on $G \times \mathcal{R}^{*}$, where $g, h: G \rightarrow \mathcal{R}^{*}$ are arbitrary functions. Put $m=\max _{t \in \mathcal{R}}|\{x \in G: g(x) h(x)=t\}|$. For any sets $\mathcal{A} \subset G$ and $\mathcal{B}, \mathcal{C} \subset \mathcal{R}^{*}$, we have

$$
|f(\mathcal{A}, \mathcal{B})||\mathcal{B} \cdot \mathcal{C}| \gg \min \left\{\frac{q^{r}|B|}{m}, \frac{|\mathcal{A}||\mathcal{B}|^{2}|\mathcal{C}|}{m^{2} q^{2 r-1}}\right\} .
$$

Key words: Finite valuation rings, sum-product estimates. 
Theorem 1.2 (Ham-Pham-Vinh, [6]). Let $\mathcal{R}$ be a finite valuation ring of order $q^{r}$, and $\mathcal{R}^{*}$ denote the set of units of $\mathcal{R}$. Let $G$ be a subgroup of $\mathcal{R}^{*}$, and $f(x, y)=g(x)(h(x)+y)$ be defined on $G \times \mathcal{R}^{*}$, where $g, h: G \rightarrow \mathcal{R}^{*}$ are arbitrary functions. Put $m=\max _{t \in \mathcal{R}}|\{x \in G: g(x)=t\}|$. For any sets $\mathcal{A} \subset G$ and $\mathcal{B}, \mathcal{C} \subset \mathcal{R}^{*}$, we have

$$
|f(\mathcal{A}, \mathcal{B})||\mathcal{B}+\mathcal{C}| \gg \min \left\{\frac{q^{r}|\mathcal{B}|}{m}, \frac{|\mathcal{A}||\mathcal{B}|^{2}|\mathcal{C}|}{m^{2} q^{2 r-1}}\right\} .
$$

Here, and throughout, $X \gg Y$ means that there exists a positive constant $c$ such that $X \geq c Y$, and " $\ll "$ is defined in a similar way. We also say that $X \sim Y$ if $X \gg Y$ and $Y \gg X$. These above results are generalizations of earlier results due to Hegyvári and Hennecart 8 in the prime field setting. A prime field version for small sets can also be found in the work on Mojarrad and Pham 9].

For any $\mathcal{A} \subset \mathcal{R}^{*}$, it follows from Theorem 1.2 with $g(x)=x$ and $h(x) \equiv 0$ that

$$
|\mathcal{A} \cdot \mathcal{A}||\mathcal{A}+\mathcal{A}| \gg \min \left\{q^{r}|\mathcal{A}|, \frac{|\mathcal{A}|^{4}}{q^{2 r-1}}\right\}
$$

Thus,

$$
\max \{|\mathcal{A}+\mathcal{A}|,|\mathcal{A} \cdot \mathcal{A}|\} \gg \min \left\{q^{\frac{r}{2}}|\mathcal{A}|^{\frac{1}{2}}, \frac{|\mathcal{A}|^{2}}{q^{\frac{2 r-1}{2}}}\right\} .
$$

For $\mathcal{A} \subset \mathcal{R}$, we define $\mathcal{A}^{2}:=\left\{x^{2}: x \in \mathcal{A}\right\}$. In a recent work, Yazici [1] studied another sum-product type estimate. Namely, she proved the following theorem.

Theorem 1.3 (Yazici, [1]). Let $\mathcal{R}$ be a finite valuation ring of $q^{r}$ and $\mathcal{A}$ be a subset of $\mathcal{R}$. If $|\mathcal{A}+\mathcal{A}||\mathcal{A}|^{2} \gg q^{3 r-1}$, then

$$
\max \left\{|\mathcal{A}+\mathcal{A}|,\left|\mathcal{A}^{2}+\mathcal{A}^{2}\right|\right\} \gg q^{\frac{r}{4}}|\mathcal{A}|^{\frac{3}{4}}
$$

It follows from Theorem 1.3 that either the size of $\mathcal{A}+\mathcal{A}$ or $\mathcal{A}^{2}+\mathcal{A}^{2}$ is large when $|\mathcal{A}|$ is big enough. Note that this sum-product type estimate was first studied by Solymosi in [16. In this paper, we provide two improvements of this result. Our first result is stated as follows.

Theorem 1.4. Let $\mathcal{R}$ be a finite valuation ring of order $q^{r}$ with $q$ a power of an odd prime number. For $\mathcal{A} \subset \mathcal{R}$ with $|\mathcal{A}| \geq 2 q^{r-1}$, then

$$
\max \left\{|\mathcal{A}+\mathcal{A}|,\left|\mathcal{A}^{2}+\mathcal{A}^{2}\right|\right\} \gg \min \left\{q^{\frac{r}{2}}|\mathcal{A}|^{\frac{1}{2}}, \frac{|\mathcal{A}|^{2}}{q^{\frac{2 r-1}{2}}}\right\} .
$$

One can check that Theorem 1.4 improves Theorem 1.3 when $|\mathcal{A}| \gg q^{r-\frac{1}{3}}$. Indeed, it is clear that

$$
\min \left\{q^{\frac{r}{2}}|\mathcal{A}|^{\frac{1}{2}}, \frac{|\mathcal{A}|^{2}}{q^{\frac{2 r-1}{2}}}\right\}=q^{\frac{r}{2}}|\mathcal{A}|^{\frac{1}{2}} .
$$

Thus, under the condition $|\mathcal{A}| \gg q^{r-\frac{1}{3}}$, the conclusion of Theorem 1.4 is stronger than that of Theorem 1.3. We also need to compare the conditions of these two theorems. Obviously, the assumption $|\mathcal{A}| \gg q^{r-\frac{1}{3}}$ implies the condition $|\mathcal{A}+\mathcal{A}||\mathcal{A}|^{2} \gg q^{3 r-1}$ by using the fact that $|\mathcal{A}+\mathcal{A}| \geq|\mathcal{A}|$. When $|\mathcal{A}+\mathcal{A}||\mathcal{A}|^{2} \gg q^{3 r-1}$ and $|\mathcal{A}| \ll q^{r-\frac{1}{3}}$, we have another improvement of Theorem 1.3, which is a consequence of the following theorem. 
Theorem 1.5. Let $\mathcal{R}$ be a finite valuation ring of order $q^{r}$ with $q$ a power of an odd prime number. For $\mathcal{A} \subset \mathcal{R}$ with $|\mathcal{A}| \geq 2 q^{r-1}$ and $|\mathcal{A}+\mathcal{A}||\mathcal{A}|^{2} \gg q^{3 r-1}$, then

$$
\max \left\{|\mathcal{A}+\mathcal{A}|,\left|\mathcal{A}^{2}+\mathcal{A}^{2}\right|\right\} \gg q^{r / 3}|\mathcal{A}|^{2 / 3} \text {. }
$$

It is convenient to give a brief comparison between these theorems. It follows from Theorem 1.4 that if $|\mathcal{A}| \ll q^{r-\frac{1}{3}}$, then $\max \left\{|\mathcal{A}+\mathcal{A}|,\left|\mathcal{A}^{2}+\mathcal{A}^{2}\right|\right\} \gg q^{\frac{1-2 r}{2}}|\mathcal{A}|^{2}$, which is better than the bound $q^{\frac{r}{4}}|\mathcal{A}|^{\frac{3}{4}}$ of Theorem 1.3 whenever $|\mathcal{A}| \gg q^{r-\frac{2}{5}}$, and weaker than the threshold $q^{r / 3}|\mathcal{A}|^{2 / 3}$ of Theorem 1.5 whenever $|\mathcal{A}| \ll q^{r-\frac{3}{8}}$. Thus, in the range $q^{r-\frac{3}{8}} \ll|\mathcal{A}| \ll q^{r-\frac{1}{3}}$, the bound $\frac{|\mathcal{A}|^{2}}{q^{\frac{2 r-1}{2}}}$ is the best. If $|\mathcal{A}+\mathcal{A}||\mathcal{A}|^{2} \gg q^{3 r-1}$ and $2 q^{r-1} \leq|\mathcal{A}| \ll q^{r-\frac{3}{8}}$, then the lower bound of Theorem 1.3 is the strongest. In other words, we can summarize the bounds in the following corollary.

Corollary 1.6. Let $\mathcal{R}$ be a finite valuation ring of order $q^{r}$ with $q$ a power of an odd prime number, and $\mathcal{A}$ be a set in $\mathcal{R}$.

1. If $|\mathcal{A}| \gg q^{r-\frac{1}{3}}$, then

$$
\max \left\{|\mathcal{A}+\mathcal{A}|,\left|\mathcal{A}^{2}+\mathcal{A}^{2}\right|\right\} \gg q^{\frac{r}{2}}|\mathcal{A}|^{\frac{1}{2}}
$$

2. If $q^{r-\frac{3}{8}} \ll|\mathcal{A}| \ll q^{r-\frac{1}{3}}$, then

$$
\max \left\{|\mathcal{A}+\mathcal{A}|,\left|\mathcal{A}^{2}+\mathcal{A}^{2}\right|\right\} \gg \frac{|\mathcal{A}|^{2}}{q^{\frac{2 r-1}{2}}} .
$$

3. If $|\mathcal{A}+\mathcal{A}||\mathcal{A}|^{2} \gg q^{3 r-1}$ and $2 q^{r-1} \leq|\mathcal{A}| \ll q^{r-\frac{3}{8}}$, then

$$
\max \left\{|\mathcal{A}+\mathcal{A}|,\left|\mathcal{A}^{2}+\mathcal{A}^{2}\right|\right\} \gg q^{r / 3}|\mathcal{A}|^{2 / 3} \text {. }
$$

Furthermore, we have a remark on the last statement of Corollary [1.6. If $|\mathcal{A}+\mathcal{A}||\mathcal{A}|^{2} \gg q^{3 r-1}$ and $|\mathcal{A}| \ll q^{r-\frac{3}{8}}$, then $|\mathcal{A}+\mathcal{A}| \gg|\mathcal{A}| q^{\frac{1}{8}}$. This leads to $\mathcal{A}+\mathcal{A}$ is an expanding set. However, the lower bound $q^{r / 3}|\mathcal{A}|^{2 / 3}$ for $\max \left\{|\mathcal{A}+\mathcal{A}|,\left|\mathcal{A}^{2}+\mathcal{A}^{2}\right|\right\}$ is stronger whenever $|\mathcal{A}| \ll q^{r-\frac{3}{8}}$. We refer the interested readers to [4, 14] for related sum-product results in the finite ring setting.

The main difference between our method and that of Yazici is that she used the Plünnecke-Ruzsa inequality. Instead of which, to prove Theorem 1.3 and Theorem 1.4, we will use spectral graph theory techniques and some ideas from the work of Pham, Vinh, and De Zeeuw [15, Theorem 1.3]. Especially, our method can be easily extended to the case of higher dimensions. In particular, we obtain the following main results which are extensions of Theorem 1.4 and Theorem 1.5, respectively. (Here, for a positive integer $n$, we use notation $n \mathcal{A}^{2}$ for the set consisting of all elements of the form $a_{1}+a_{2}+\cdots+a_{n}$ with $a_{1}, a_{2}, \ldots, a_{n}$ in $\mathcal{A}^{2}$.)

Theorem 1.7. Let $\mathcal{R}$ be a finite valuation ring of order $q^{r}$ with $q$ a power of an odd prime number. For any $\mathcal{A} \subset \mathcal{R}$ with $|\mathcal{A}| \geq 2 q^{r-1}$ and for any integer $n>1$, we have

$$
\max \left\{\left|n \mathcal{A}^{2}\right|,|\mathcal{A}+\mathcal{A}|\right\} \gg \min \left\{q^{\frac{r}{n}}|\mathcal{A}|^{\frac{n-1}{n}}, \frac{|\mathcal{A}|^{\frac{3 n-2}{n}}}{q^{\frac{(n-1)(2 r-1)}{n}}}\right\} .
$$

Theorem 1.8. Let $\mathcal{R}$ be a finite valuation ring of order $q^{r}$ with $q$ a power of an odd prime number. For any $\mathcal{A} \subset \mathcal{R}$ with $|\mathcal{A}| \geq 2 q^{r-1}$ and for any integer $n>1$, we have

$$
\max \left\{|\mathcal{A}+\mathcal{A}|,\left|n \mathcal{A}^{2}\right|\right\} \gg q^{\frac{r}{2 n-1}}|\mathcal{A}|^{\frac{2 n-2}{2 n-1}},
$$

whenever $|\mathcal{A}+\mathcal{A}|^{n-1}|\mathcal{A}|^{n} \gg q^{r+(n-1)(2 r-1)}$.

In the rest of the paper, we are going to give proofs of Theorem 1.7 and Theorem 1.8 


\section{The definition of finite valuation rings}

We start this section by recalling the definition of finite valuation rings from [10].

A commutative ring with identity is called a finite valuation ring if it is finite, local and principal.

Let $\mathcal{R}$ be a finite valuation ring. We have that $\mathcal{R}$ contains a unique maximal ideal, denoted by $(z)$ for some non-unit uniformizer element $z$ in $\mathcal{R}$. Notice that the uniformizer element is defined up to a unit in $\mathcal{R}$. We also denote by $\mathcal{R}^{*}, \mathcal{R}^{0}$ the set of units, non-units in $\mathcal{R}$, respectively.

Since $(z)$ is the maximal ideal, we have $\mathcal{R} /(z)$ is a field, which is denoted by $F$. We denote the size of $F$ by $q$. Let $r$ be the nilpotency degree of $z$, i.e. the smallest positive integer $r$ satisfying $z^{r}=0$. It is known that $q$ is a power of a prime number. In this paper, we assume that $q$ is odd, so 2 is a unit in $\mathcal{R}$, i.e $2 \in \mathcal{R}^{*}$.

Over finite valuation rings $\mathcal{R}$, one has a natural valuation

$$
f: \mathcal{R} \rightarrow\{0,1, \ldots, r\}
$$

defined by $f(0)=r$ and for $x \neq 0, f(x)=k$ if $x \in\left(z^{k}\right) \backslash\left(z^{k+1}\right)$. This means that, $f(x)=k$ if and only if $x=u z^{k}$ for some unit $u$ in $\mathcal{R}$. One can check that for each $k$, the group group $\left(z^{k}\right) /\left(z^{k+1}\right)$ is a one-dimensional linear space over the residue field $F=\mathcal{R} /(z)$, thus its size is $q$. Hence, $\left|\left(z^{k}\right)\right|=q^{r-k}$ for $k=0,1, \ldots, r$. In other words, we have $|(z)|=q^{r-1},|\mathcal{R}|=q^{r}$ and $\left|\mathcal{R}^{*}\right|=|\mathcal{R}|-|(z)|=q^{r}-q^{r-1}$. We refer the readers to [10] for more details. There are several examples of finite valuation rings, for instance, finite fields $\mathbb{F}_{q}$ with $q$ is a prime power, finite cyclic rings $\mathbb{Z} / p^{r} \mathbb{Z}$ with $p$ is a prime, and $\mathcal{O} /\left(p^{r}\right)$, where $\mathcal{O}$ is the ring of integers in a number field and $p \in \mathcal{O}$ is a prime.

\section{Techniques from spectral graph theory}

Let $G=(A \cup B, E)$ be a bipartite graph. If all vertices in each part have the same degree, we say that $G$ is biregular. If $G$ is biregular, we denote the common degree of each vertex in $A$ by $\operatorname{deg}(A)$, and the degree of each vertex in $B$ by $\operatorname{deg}(B)$. Let $M$ be the adjacency matrix of $G$. Assume that $\lambda_{1}, \ldots, \lambda_{n}$ are eigenvalues of $M$ with $\left|\lambda_{1}\right| \geq\left|\lambda_{2}\right| \geq \cdots \geq\left|\lambda_{n}\right|$. Since $G$ is a bipartite graph, we have $\lambda_{2}=-\lambda_{1}$. To prove our main theorems, we will make use of the following version of the Expander mixing lemma for bipartite graphs. We refer the readers to [5] for a proof.

Lemma 3.1. Suppose that $G=(A \cup B, E)$ is biregular with $\operatorname{deg}(A)=a$ and $\operatorname{deg}(B)=b$. For subsets $X \subset A$ and $Y \subset B$, the number of edges between $X$ and $Y$, denoted by $e(X, Y)$, satisfies

$$
\left|e(X, Y)-\frac{a}{|B|}\right| X|| Y|| \leq \lambda_{3} \sqrt{|X||Y|}
$$

where $\lambda_{3}$ is the third eigenvalue of $G$. We note that $a /|B|=b /|A|$.

For any $\boldsymbol{x} \in \mathcal{R}^{d} \backslash\left(\mathcal{R}^{0}\right)^{d}$, denote by $[\boldsymbol{x}]$ the equivalence class of $\boldsymbol{x}$ in $\mathcal{R}^{d} \backslash\left(\mathcal{R}^{0}\right)^{d}$, where $\boldsymbol{x}, \boldsymbol{y} \in \mathcal{R}^{d} \backslash\left(\mathcal{R}^{0}\right)^{d}$ are in the same class iff $\boldsymbol{x}=\boldsymbol{t} \boldsymbol{y}$ for some unit $t \in \mathcal{R}^{*}$. Let $\mathcal{E}_{q, d}(\mathcal{R})=(A \cup B, E)$ be the Erdös-Rényi bipartite graph with $A$ and $B$ being the sets of equivalence classes in $\mathcal{R}^{d} \backslash\left(\mathcal{R}^{0}\right)^{d}$. There is an edge between two vertices $[\boldsymbol{x}]$ and $[\boldsymbol{y}]$ iff $\boldsymbol{x} \cdot \boldsymbol{y}=0$. The spectrum of this graph over finite valuation rings was studied by Nica [10] by using exponential sums. We summary in the following theorem.

Theorem 3.2 (Nica, [10]). The cardinality of each vertex part of $\mathcal{E}_{q, d}(\mathcal{R})$ is $q^{(d-1)(r-1)}\left(q^{d}-1\right) /(q-$ $1)$, and $\operatorname{deg}(A)=\operatorname{deg}(B)=q^{(d-2)(r-1)}\left(q^{d-1}-1\right) /(q-1)$. The third eigenvalue of $\mathcal{E}_{q, d}(\mathcal{R})$ is at most $\sqrt{q^{(d-2)(2 r-1)}}$. 


\section{Proof of Theorem 1.7}

Since $|\mathcal{A}| \geq 2 q^{r-1}$, it follows that

$$
\left|\mathcal{A} \cap \mathcal{R}^{*}\right| \geq|\mathcal{A}|-\left|\mathcal{R}^{0}\right|=|\mathcal{A}|-q^{r-1} \geq \frac{|\mathcal{A}|}{2} .
$$

Thus we may assume that $\mathcal{A}$ is a subset of $\mathcal{R}^{*}$. We now prove that the size of the set $\mathcal{A}^{2}$ is at least $\gg|\mathcal{A}|$. Indeed, suppose $x^{2}=y^{2}$ with $x, y \in A$, then $(x-y)(x+y)=0$. There are posibilities for pairs $(x, y)$ as follows: $x=y$, or $x=-y$, or $x-y \in(z)$ and $x+y \in(z)$. If the last case holds, then we can write $x-y=u_{1} z_{1}^{k}$ and $x+y=u_{2} z^{k_{2}}$ with $u_{1}, u_{2} \in \mathcal{R}^{*}$ and some positive integers $k_{1}, k_{2}$. This leads to $2 x=u_{1} z^{k_{1}}+u_{2} z^{k_{2}} \in(z)$, which gives a contradiction since both 2 and $x$ are in $\mathcal{R}^{*}$. In other words, either $x=y$ or $x=-y$ and therefore $\left|\mathcal{A}^{2}\right| \gg|\mathcal{A}|$.

Define $\mathcal{D}=n \mathcal{A}^{2}$. Consider the following equation

$$
x+\left(b_{1}-c_{1}\right)^{2}+\cdots+\left(b_{n-1}-c_{n-1}\right)^{2}=t,
$$

where $x \in \mathcal{A}^{2}, b_{i} \in \mathcal{A}+\mathcal{A}, c_{i} \in \mathcal{A}, 1 \leq i \leq n-1, t \in D$. Let $N$ be the number of solutions of this equation. We first see that $N \geq|\mathcal{A}|^{2 n-1}$. Let $U$ and $V$ be two vertex sets of the Erdös-Rényi graph $\mathcal{E}_{q, n+1}(\mathcal{R})$ defined by

$$
U:=\left\{\left(-2 b_{1}, \ldots,-2 b_{n-1}, \sum_{i=1}^{n-1} b_{i}^{2}+x, 1\right): b_{i} \in \mathcal{A}+\mathcal{A}, 1 \leq i \leq n-1, x \in \mathcal{A}^{2}\right\},
$$

and

$$
V:=\left\{\left(c_{1}, \ldots, c_{n-1}, 1, \sum_{i=1}^{n-1} c_{i}^{2}-t\right): c_{i} \in \mathcal{A}, 1 \leq i \leq n-1, t \in \mathcal{D}\right\} .
$$

We have $|U| \sim|\mathcal{A}+\mathcal{A}|^{n-1}|\mathcal{A}|$ and $|V|=|\mathcal{A}|^{n-1}|\mathcal{D}|$. It is not hard to check that $N$ is bounded by the number of edges between $U$ and $V$ in the graph $\mathcal{E}_{q, n+1}(\mathcal{R})$. Thus, one can apply Lemma 3.1 and Theorem 3.2 to get

$$
N \ll \frac{|U||V|}{q^{r}}+q^{\frac{(n-1)(2 r-1)}{2}} \sqrt{|U||V|}=\frac{|\mathcal{A}+\mathcal{A}|^{n-1}|\mathcal{A}|^{n}\left|n \mathcal{A}^{2}\right|}{q^{r}}+q^{\frac{(n-1)(2 r-1)}{2}} \sqrt{|\mathcal{A}+\mathcal{A}|^{n-1}|\mathcal{A}|^{n}\left|n \mathcal{A}^{2}\right|} .
$$

Using the fact that $N \geq|\mathcal{A}|^{2 n-1}$, we obtain

$$
\max \left\{\left|n \mathcal{A}^{2}\right|,|\mathcal{A}+\mathcal{A}|\right\} \gg \min \left\{q^{\frac{r}{n}}|\mathcal{A}|^{\frac{n-1}{n}}, \frac{|\mathcal{A}|^{\frac{3 n-2}{n}}}{q^{\frac{(n-1)(2 r-1)}{n}}}\right\} .
$$

This completes the proof of the theorem.

\section{Proof of Theorem 1.8}

Since $|\mathcal{A}| \geq 2 q^{r-1}$, as in the proof of Theorem [1.7, we may assume that $\mathcal{A}$ is a subset of $\mathcal{R}^{*}$. In this proof, we will follow the idea of [15, Theorem 1.3].

As in the proof of Theorem [1.7, we define $D=n \mathcal{A}^{2}$. Consider the following equation

$$
x+\left(b_{1}-c_{1}\right)^{2}+\cdots+\left(b_{n-1}-c_{n-1}\right)^{2}=t,
$$

where $x \in \mathcal{A}^{2}, b_{i} \in \mathcal{A}+\mathcal{A}, c_{i} \in \mathcal{A}, 1 \leq i \leq n-1, t \in D$. Let $N$ be the number of solutions of this equation. We see that $N \geq|\mathcal{A}|^{2 n-1}$. 
By the Cauchy-Schwarz inequaltiy, one has

$$
N^{2} \leq|\mathcal{D}| \cdot E,
$$

where $E$ is the number of tuples $\left(x, b_{1}, \ldots, b_{n-1}, c_{1}, \ldots, c_{n-1}, y, d_{1}, \ldots, d_{n-1}, e_{1}, \ldots, e_{n-1}\right)$ satisfying

$$
x+\left(b_{1}-c_{1}\right)^{2}+\cdots+\left(b_{n-1}-c_{n-1}\right)^{2}=y+\left(d_{1}-e_{1}\right)^{2}+\cdots+\left(d_{n-1}-e_{n-1}\right)^{2} .
$$

Let $U$ and $V$ be two vertex sets in the Erdős-Rényi graph $\mathcal{E}_{q, 2 n}(\mathcal{R})$ defined by

$$
U:=\left\{\left(-2 b_{1}, \ldots,-2 b_{n-1}, 2 d_{1}, \ldots, 2 d_{n-1}, 1, \sum_{i=1}^{n-1} b_{i}^{2}-\sum_{i=1}^{n-1} d_{i}^{2}+x\right): b_{i} \in \mathcal{A}+\mathcal{A}, d_{i} \in \mathcal{A}, x \in \mathcal{A}^{2}\right\},
$$

and

$$
V:=\left\{\left(c_{1}, \ldots, c_{n-1}, e_{1}, \ldots, e_{n-1}, \sum_{i=1}^{n-1} c_{i}^{2}-\sum_{i=1}^{n-1} e_{i}^{2}-y, 1\right): c_{i} \in \mathcal{A}, e_{i} \in \mathcal{A}+\mathcal{A}, y \in \mathcal{A}^{2}\right\} .
$$

We have $|U|=|V| \sim|\mathcal{A}+\mathcal{A}|^{n-1}|\mathcal{A}|^{n}$. We also have $E$ is bounded by the number of edges between $U$ and $V$ in the graph $\mathcal{E}_{q, 2 n}(\mathcal{R})$. Therefore, it follows from Lemma 3.1 and Theorem 3.2 that

$$
E \ll \frac{|\mathcal{A}+\mathcal{A}|^{2 n-2}|\mathcal{A}|^{2 n}}{q^{r}}+q^{(n-1)(2 r-1)}|\mathcal{A}+\mathcal{A}|^{n-1}|\mathcal{A}|^{n} .
$$

Using the facts that $N \geq|\mathcal{A}|^{2 n-1}$ and $N^{2} \leq|\mathcal{D}| \cdot E$, we derive

$$
\max \left\{|\mathcal{A}+\mathcal{A}|,\left|n \mathcal{A}^{2}\right|\right\} \gg q^{\frac{r}{2 n-1}}|\mathcal{A}|^{\frac{2 n-2}{2 n-1}},
$$

whenever $|\mathcal{A}+\mathcal{A}|^{n-1}|\mathcal{A}|^{n} \gg q^{r+(n-1)(2 r-1)}$. In particular,

$$
\max \left\{|\mathcal{A}+\mathcal{A}|,\left|n \mathcal{A}^{2}\right|\right\} \gg \min \left\{q^{\frac{r}{2 n-1}}|\mathcal{A}|^{\frac{2 n-2}{2 n-1}}, \frac{|\mathcal{A}|^{\frac{3 n-2}{n}}}{q^{\frac{(n-1)(2 r-1)}{n}}}\right\},
$$

which ends the proof of the theorem.

\section{References}

[1] E. Aksoy Yazici, Sum-product type estimates for subsets of finite valuation rings, Acta Arith. 185(1) (2018), 9-18.

[2] E. Aksoy Yazici, B. Murphy, M. Rudnev, I. Shkredov, Growth estimates in positive characteristic via collisions, International Mathematics Research Notices, 2017(23), 7148-7189.

[3] P. Erdős and E. Szemerédi, On sums and products of integers, Studies in Pure Mathematics. To the memory of Paul Turán, Basel: Birkhäuser Verlag, pp. 213-218, 1983.

[4] J. Bourgain, A. Gamburd, P. Sarnak Affine linear sieve, expanders, and sum-product, Invent. Math. 179 (2010), 559-644.

[5] A. Eustis, Hypergraph Independence Numbers, PhD thesis 2013, University of California, San Diego.

[6] L. Q. Ham, T. Pham, L. A. Vinh, Conditional expanding bounds for two-variable functions over finite valuation rings, European J. Combin. 60 (2017), 114-123.

[7] M. Z. Garaev, The sum-product estimate for large subsets of prime fields, Proc. Amer. Math. Soc. 136 (2008), 2735-2739. 
[8] N. Hegyvári, F. Hennecart, Conditional expanding bounds for two-variable functions over prime fields, European J. Combin. 34(2013), 1365-1382.

[9] H. Mojarrad, T. Pham, Conditional expanding bounds for two-variable functions over arbitrary fields, Journal of Number Theory 186 (2018), 137-146.

[10] B. Nica, Unimodular graphs and Eisenstein sums, Journal of Algebraic Combinatorics 45(2) (2017), 114-123.

[11] M. Rudnev, I. Shkredov, S. Stevens, On an energy variant of the sumproduct conjecture, arXiv: 1607.05053 (2016).

[12] M. Rudnev, G. Shakan, I. Shkredov, Stronger sum-product inequalities for small sets, Proc. Amer. Math. Soc. 148 (2020), 1467-1479

[13] T. Pham, A sum-product theorem in matrix rings over finite fields, Comptes Rendus Mathematique 357(10) (2019), 776-770.

[14] T. Pham, L. A. Vinh, Erdős-Rényi graph, Szemerédi-Trotter type theorem, and sum-product estimates over finite rings, Forum Math. 27(1) (2015), 331-342.

[15] T. Pham, L. A. Vinh, F. De Zeeuw, Three-variable expanding polynomials and higher dimensional distinct distances, Combinatorica (2017), 1-16.

[16] J. Solymosi, Incidences and the spectra of graphs, Combinatorial Number Theory and Additive Group Theory, Birkhauser Basel, 2009. 299-314.

[17] C-Y, Shen, Algebraic methods in sum-product phenomena, Israel Journal of Mathematics 188(1) (2012), 123-130.

[18] V. Vu, Sum-product estimates via directed expanders, Math. Res. Lett. 15 (2008), no.2, 375388.

DuC HieP Pham

University of Education

Vietnam National University, Hanoi

144 Xuan Thuy, Cau Giay, Hanoi

Vietnam

Email: phamduchiep@vnu.edu.vn 